\title{
Occurrence of Freshwater Algae in Vena River in Hinganghat area of Dist. Wardha
}

\author{
B. M. Rajurkar ${ }^{1}$, L. P. Dalal ${ }^{2}$ \\ ${ }^{1}$ Assistant Professor, Department of Botany, R. S. Bidkar College, Hinganghat, Dist. Wardha \\ ${ }^{2}$ Associate Professor, Department of Botany, J. B. College of Science, Wardha (M.S.)
}

Abstract: The present study reports on community structure of freshwater algal taxa of Vena river in Hingan@jat arga, of Wardha District. Total 4 different stations were sampled from June 2011 to May 2013. One hundred eighteen algal taxa ere idgtified of which 73 taxa belonged to Chlorophyceae, 24 to Bacillariophyceae, 13 to Cyanophyceae and 8 to Euglenophycqae. Maymum number of species was recorded in 104 to Underbridge $\left(S W_{1}\right)$ followed by 79 to Shahalangadi $\left(S W_{4}\right), 72$ to Smaskahblogmi $\left(S W_{3}\right)$ and 68 tad Kawalghat $\left(\mathrm{SW}_{2}\right)$.

Keywords: Algae; Aquatic ecosystem; Eutrophication; Vena river

\section{Introduction}

The present research enables a comprehens and systematic analysis of the algal flora of the Kena river in $\gamma^{5}$ Hinganghat area of Wardha district, whickns a part Vidarbha, Maharashtra state for two years of Intensive stoty i.e. June 2011 to May 2013. Algae o ocurs in stepient quantities to render its commercial aphications, is been investigated by Marathe, (1969). am Jawale an ehaudhari (2010).

Hinganghat is one of tha ishils of W Districtostater

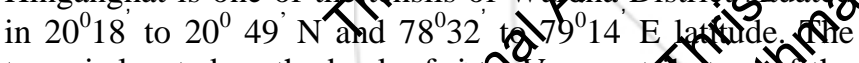
town is located on the bank of rinena, a trutary the Wardha river which joins the 30 river Prafita $20 \mathrm{~d}$ at a distance place, which ultinftely merges Sto 18 Godavari river later. In British India, Hingangh was the centre India, but after the partition of Hind a pinto Indig) and Pakistan, Nagpur is considered of genter (heart sece) India. At Vena river pump liverse, trere is a hisjoricalopt stone, on which it was metions that Hing centre of India.

Major portion of the months of Jupofor seger of every rainfall of Higangat Tahsil is 1071. and has a dry tropical climateân hot, and dry. Max temp. in noted as $47.9{ }^{\circ} \mathrm{C}$ (pti Min. temp. in ${ }^{0} \mathrm{C}$ were note 0 as \$.2 ${ }^{0} \mathrm{C}$. The seas 10 of a year were divided ofordias limates into thres 9 mely cold, hot and monsoon. He lod scape of the citaces towards the south with fast ryvang streams. Venghiver borders the north, west, and ofth sides of the chy. The city is rich in fauna, and flora and water sourcel

In Hinganghat area, Vena river is a fresh water body, and is one of the prominent river of Vidarbha, Maharashtra. It. is Perennial River of this area. It is supposed to be the life line of the Wardha district, but due to expanding needs of growing population, it is faces many adversities or changes.

the sajentific appraat yas not holistic. The

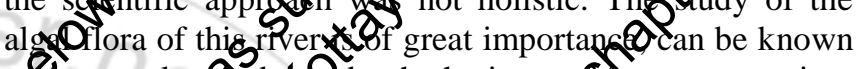
o the peoples ond wo be the heritage of uture generation. SHence, it is a neep, of hour to know efoh/and every thing of

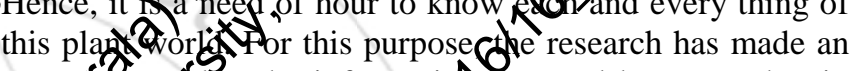
attery to the informatip reported by researcher in past.
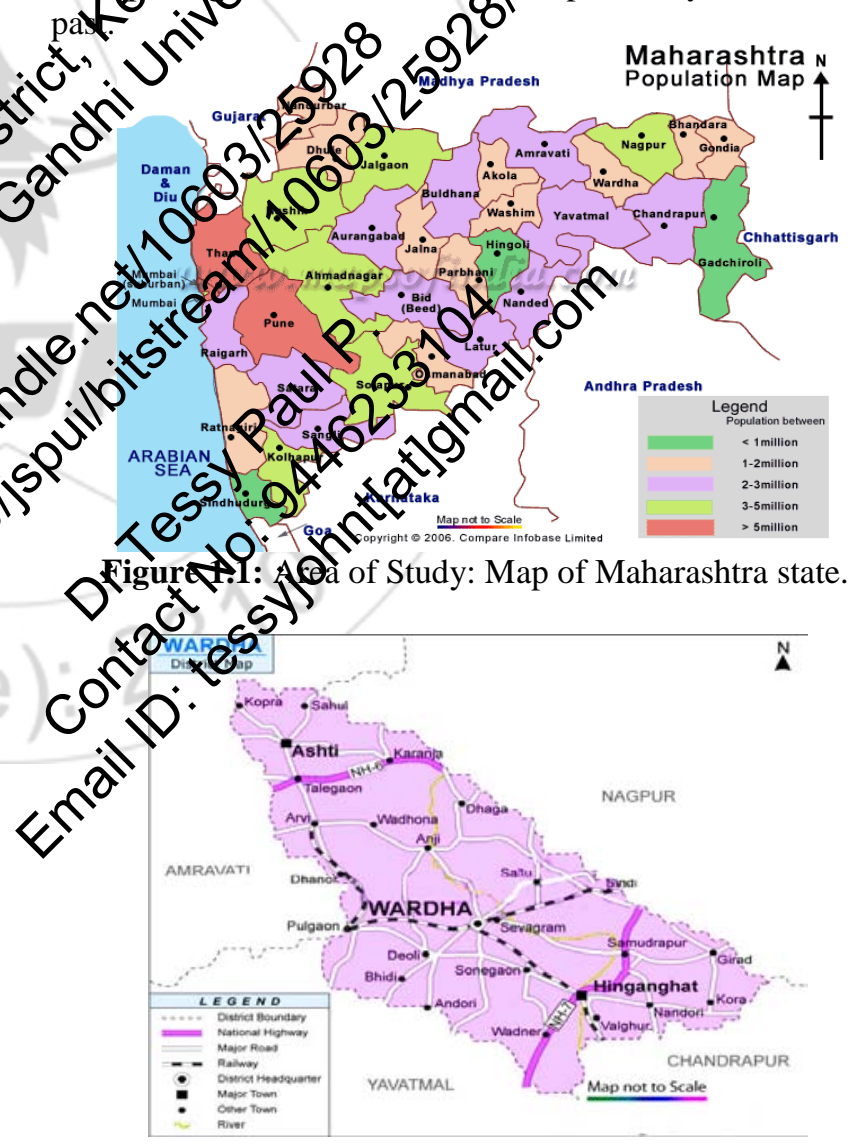

Figure 1.2: Area of Study: Map of Wardha district.

The river Vena has received little attention from botanists, ecologists and specially phycologist as such and moreover, 
International Journal of Science and Research (IJSR)

ISSN (Online): 2319-7064

Index Copernicus Value (2013): 6.14 | Impact Factor (2014): 5.611

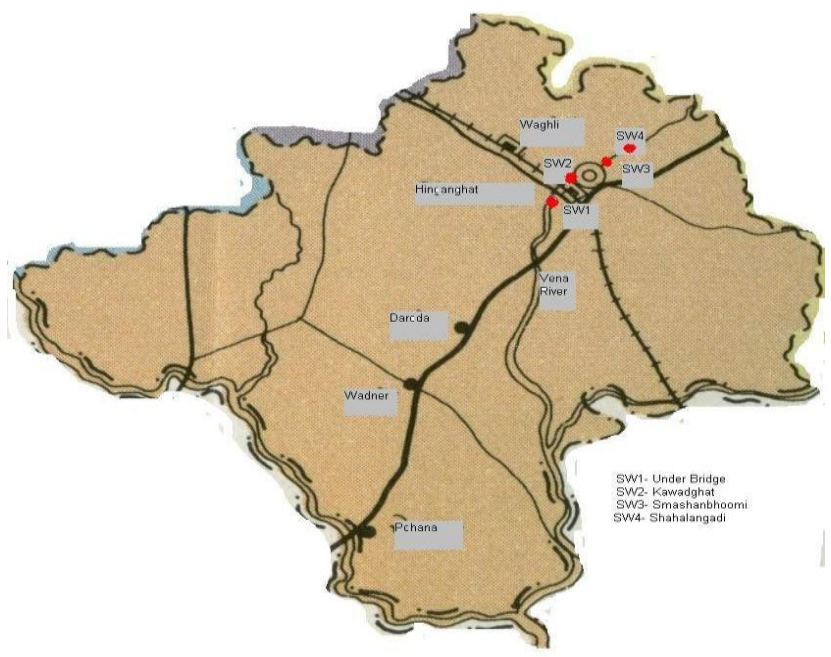

Figure 1.3: Area of Study: Map of Hinganghat Tahsil showing study area

\section{Materials and Methods}

Vena River is one of the major water bodies of Hinganghat region of Wardha District, Vidarbha. Stations $\mathrm{SW}_{1}$ (Underbridge), $\quad \mathrm{SW}_{2} \quad$ (Kawalghat), $\quad \mathrm{SW}_{3}$ (Smashanbhoomi), and $\mathrm{SW}_{4}$ (Shahalangadi) were selected near Hinganghat area. Water samples were collected from June 2011 to May 2013. These samples were analysed for determining the algal taxa. The macroscopic algadwere manually picked with forceps and microscopic dae with

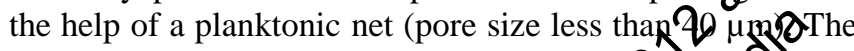
samples were immediately brought to the boratop or the taxonomical documentation of algal tax akd preserved in $4 \%$ formalin for reference purpose. ProTveg\&mples were studied after the proper settlement the samples were examined under binbculgr microscope with attached MIPS for the identiQatignof algal groups nin photographs were taken. Als ideretication was carnjல

with the help of availablexonomic literature.
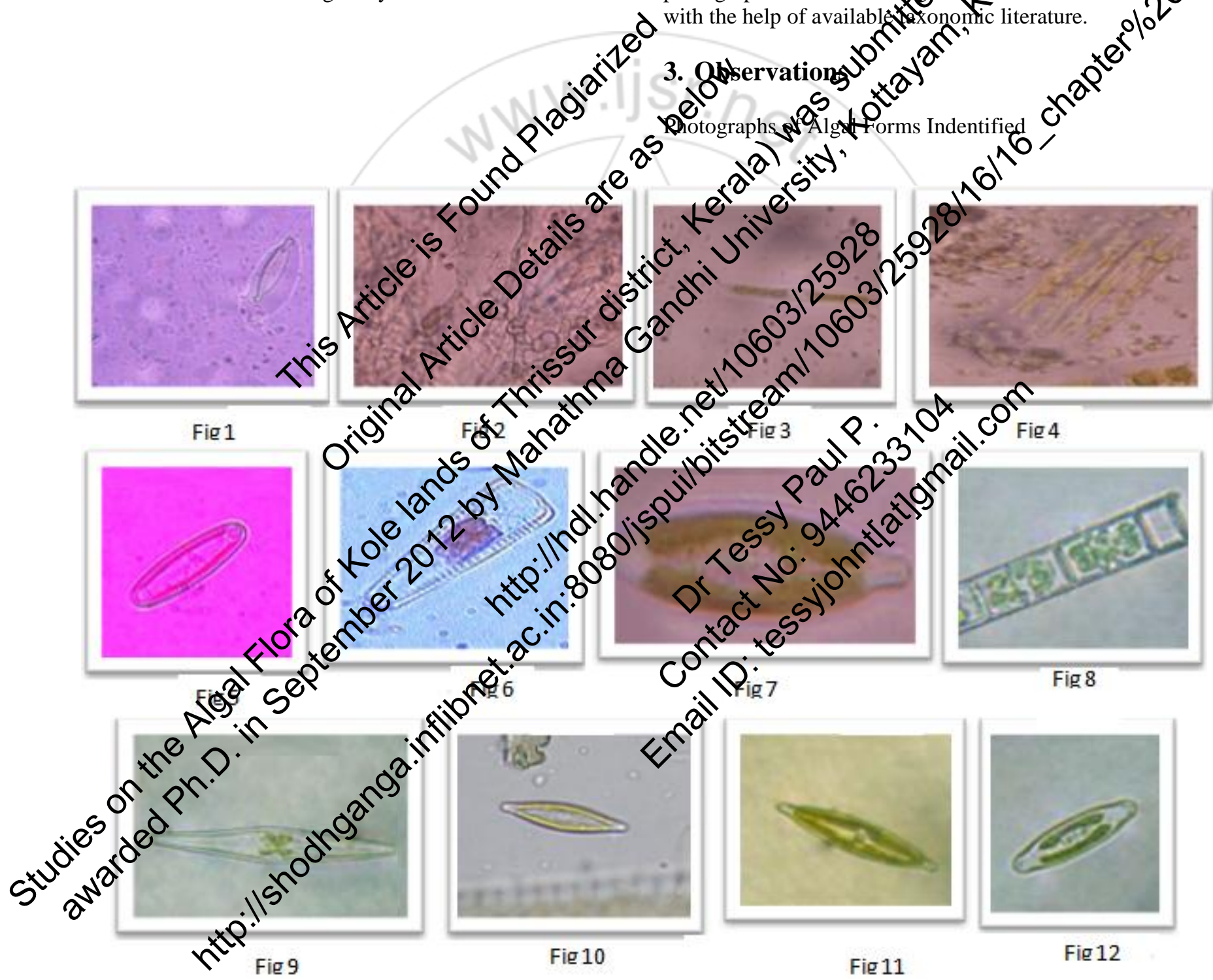

Volume 5 Issue 3, March 2016 


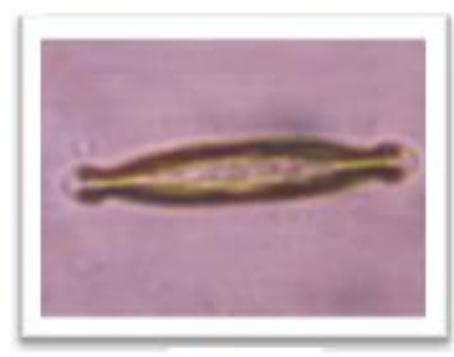

Fig 13

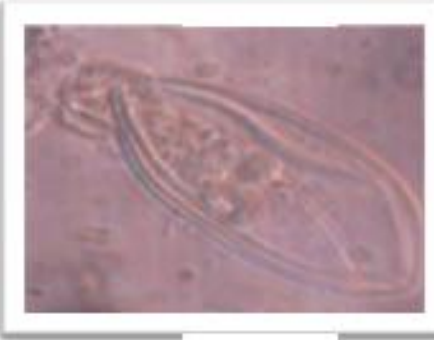

Fig 14

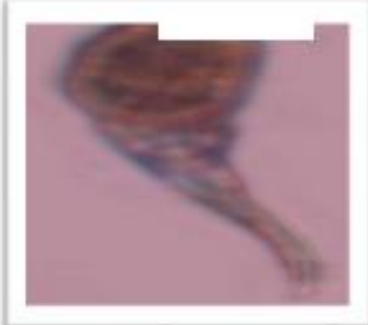

Fig 15

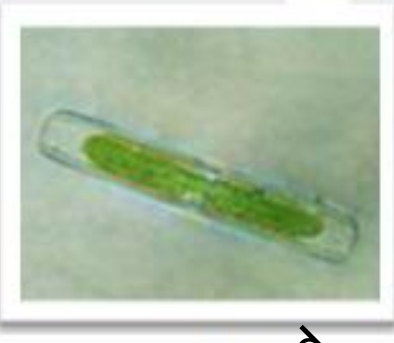

Fig

Baccilariophyceae: Fig. 1. Cymbella cistula (Hemprich) Grun. var. woosangisisVirget Fig. 2 Dinobryon sertulqriEhkeberg Fig. 3 Eunotia camelus Ehr. var. karveerensis Gandhi Fig. 4 Fragilaria virescens Ralfs Fig. 5 Frustulia rhom Dides (\$r) De Toni var. saxonica (Rabenhorst) DeToni Fig. 6 Gomphonema elegans Grun Fig. 7 Gomphonema vidarbhenswKamath Fig. 8 Melosira granulata (Ehr.) Ralfs. Fig. 9 Navicula cari Ehr. fa. indica Sarode et Kamat. Fig. 10 Navicula OPpto \&hiala Kuetz Fig. 11 Navicula cuspidata Kuetz. var.ambigua (Ehr.) Cleve. Fig. 12 Navicula pupula Kuetz. var. qqpiratg 3 Stedt. Fig. 13

Navicula viridula Kuetzing Fig. 14 Peridinium cinctum (Muller) Ehrenberg Swirenko Fig. 15 Phgcks çydatus var. tenuis

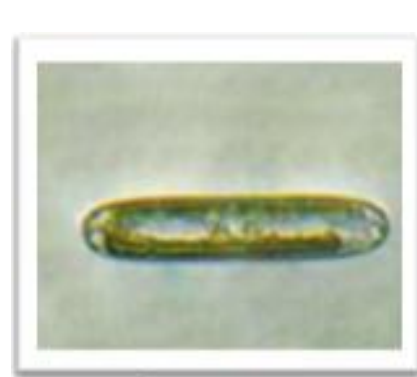

Fig. 16 Pinnularia acrosphaeria (Breb.)W.Smith

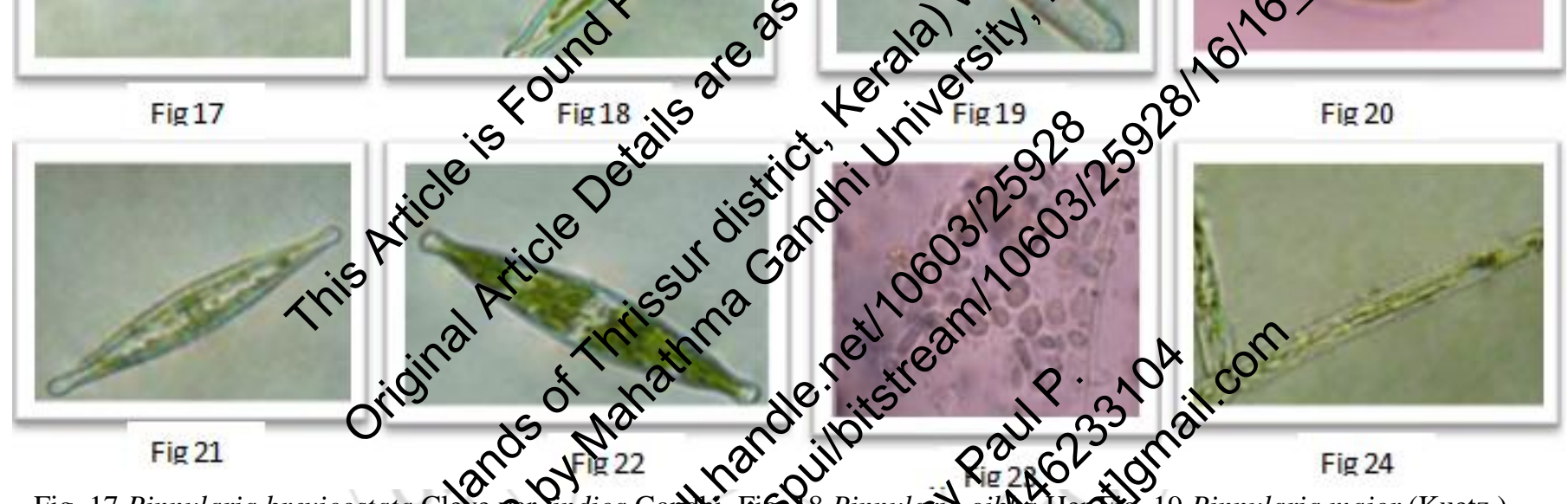

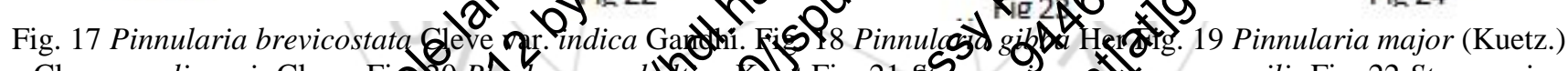

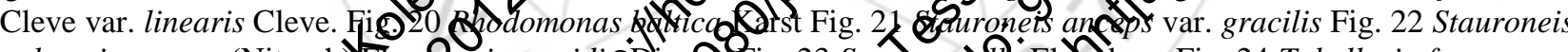

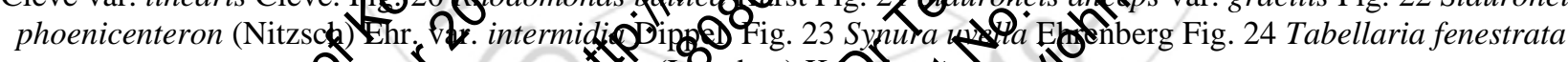

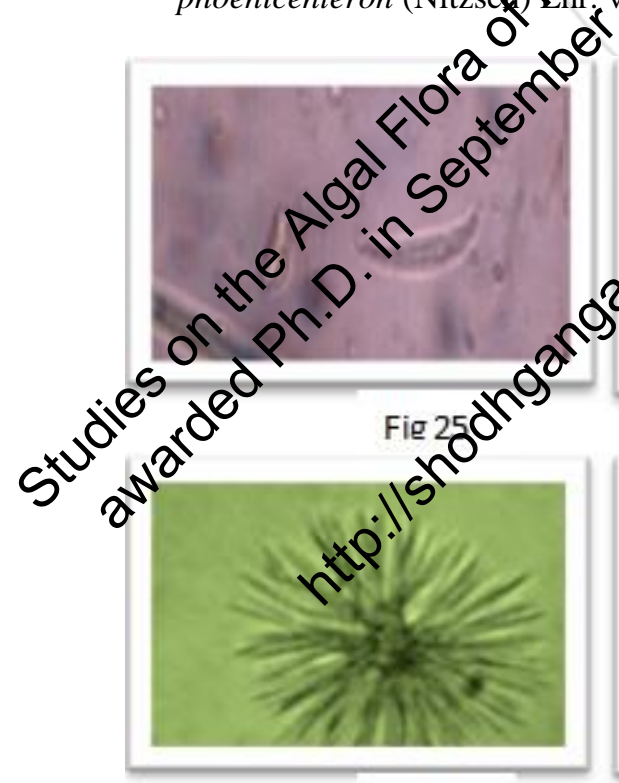

Fig 29

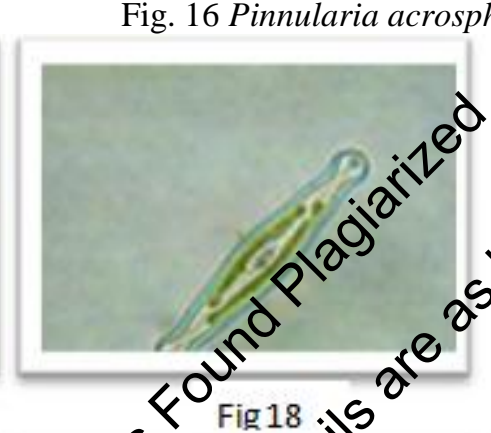

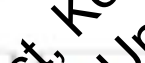
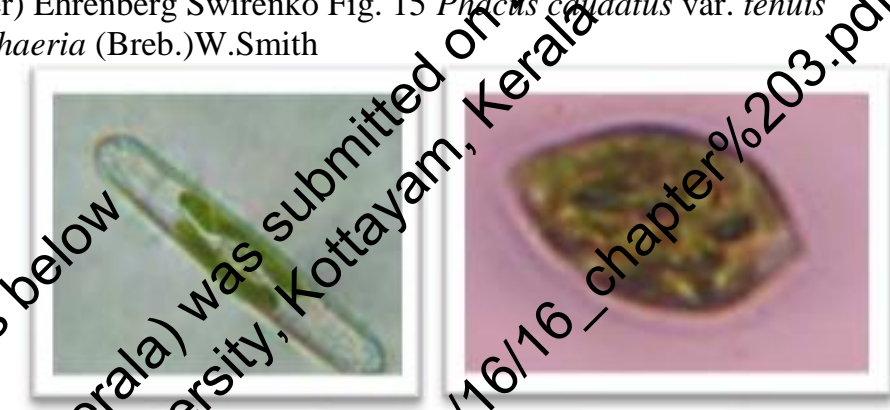

Fig 20 


\section{International Journal of Science and Research (IJSR) \\ ISSN (Online): 2319-7064 \\ Index Copernicus Value (2013): 6.14 | Impact Factor (2014): 5.611}

Braun) G.S.West Fig. 29 Ankistrodesmus spiralis (Turner) Lemm. Fig. 30 Chlorella vulgaris Beyer. (Smith) Fig. 31

Coelastrum cambricum Archer var. intermidium (Bohlin) G.S.West Fig. 32 Coelastrum sphaerium Naeg.

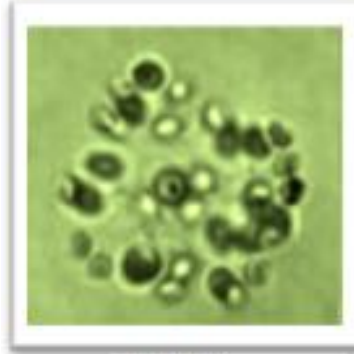

Fig 33

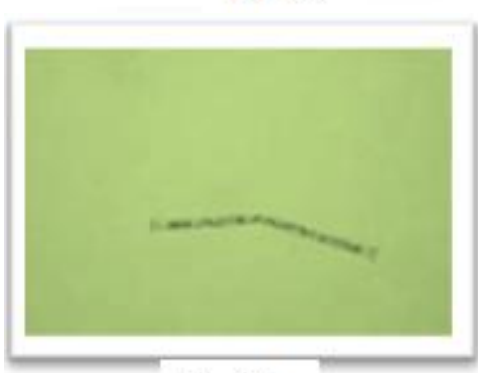

Fig 37

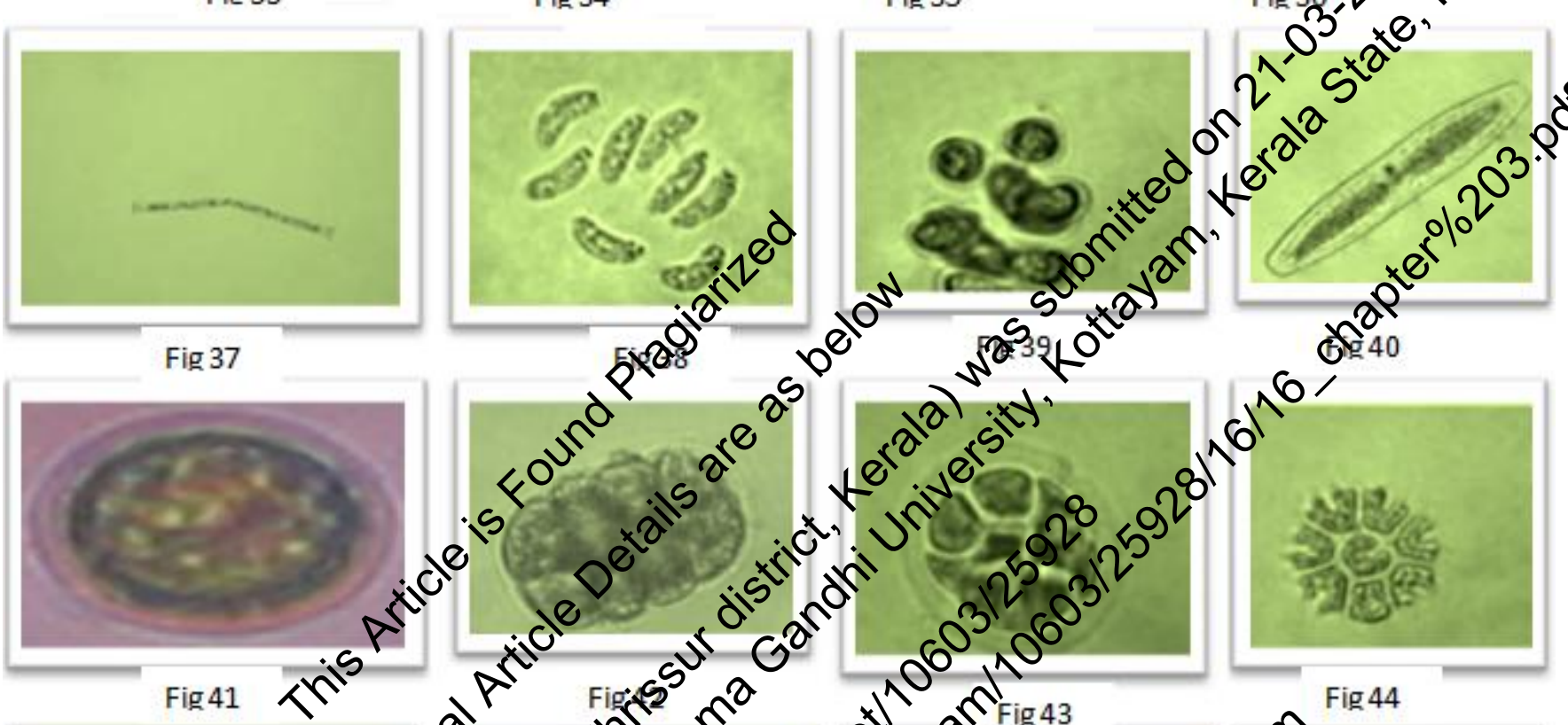

Fig 41

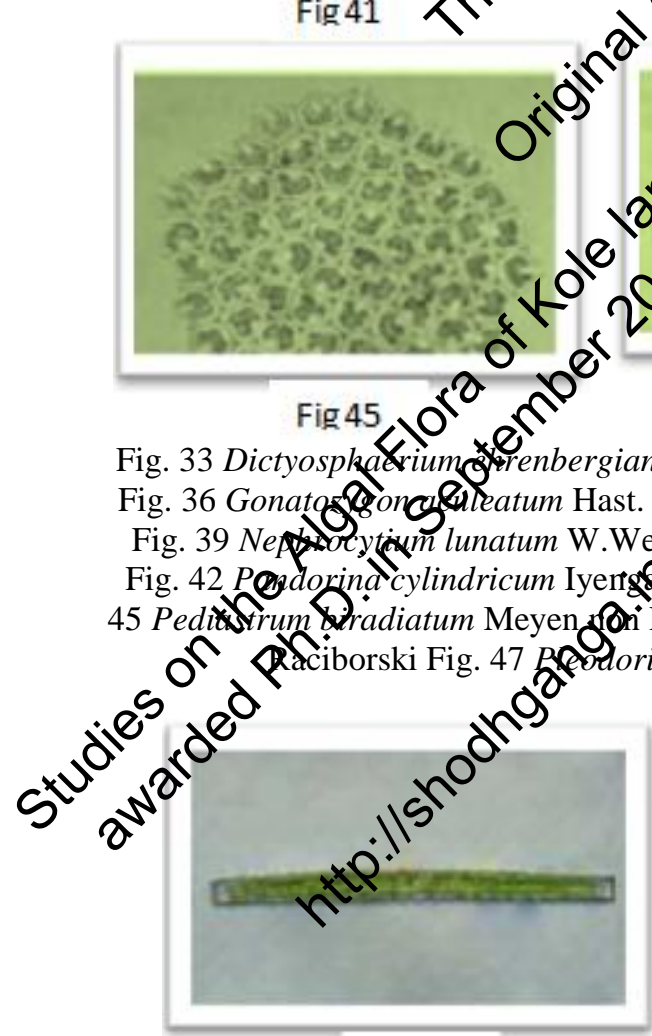

Fig 49

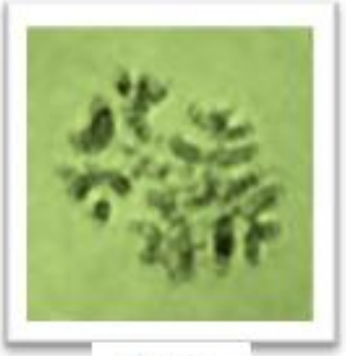

Fig 34

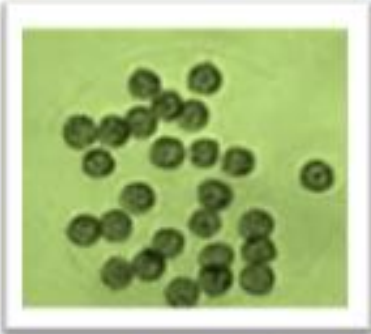

Fig 35

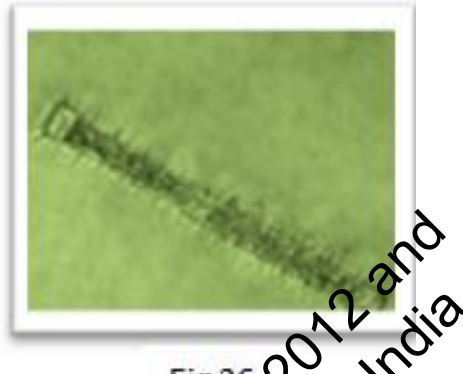

Fig 36,
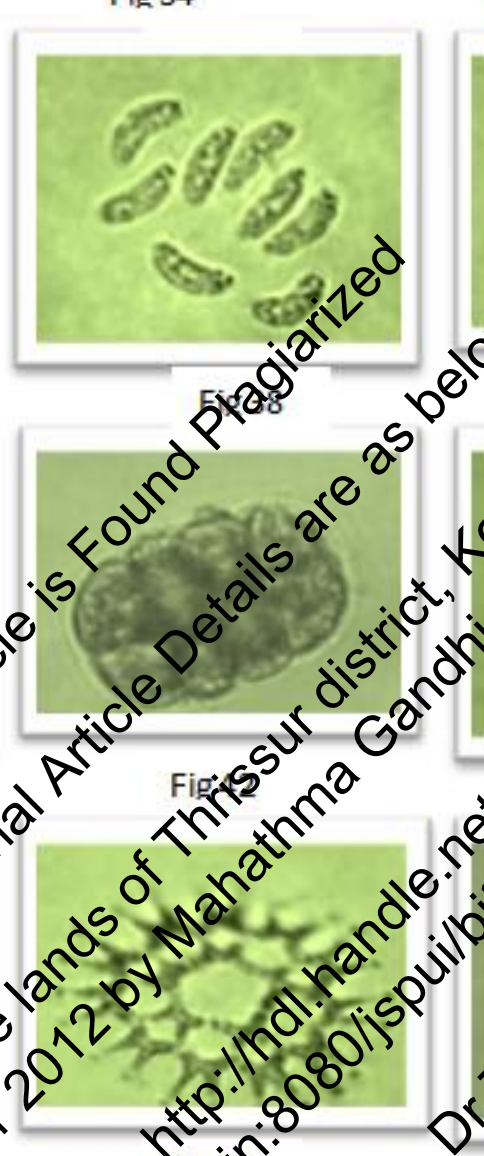

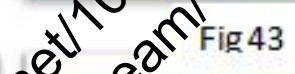

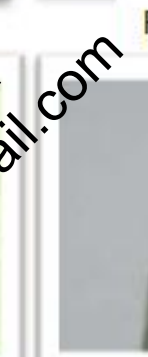

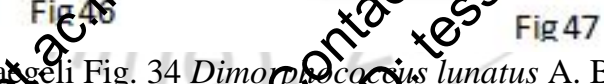

Fig 48

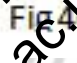

(ic

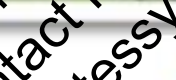

Gonatozygon

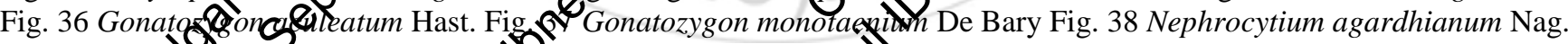
Fig. 39 Ne 1 lunatum W.We 40 Netrium digitus (EOSg.) Itzigs. \&Rothe Fig. 41 Oocystis elliptica W. West

Fig. 42 Poddorind cylindricum Iyeng Fig. 43 Pandorina. men (Mull.) Bory Fig. 44 Pediastrum tetras (Ehr.) Ralfs Fig. 45 Pedirum Sudiatum Meyen Rori Ralfs var. longicornutum Gutwinski Fig. 46 Pediastrum duplex Meyen var. coronatum

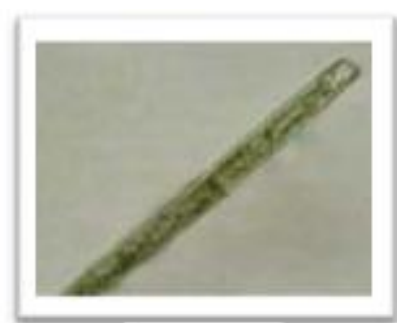

Fig 50

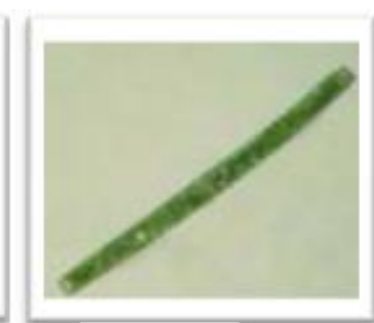

Fig 51

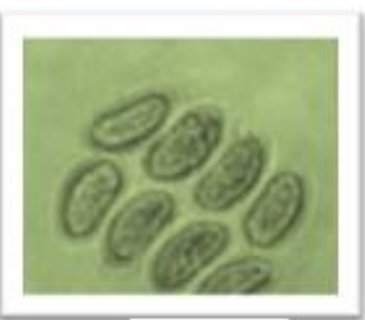

Fig 52 

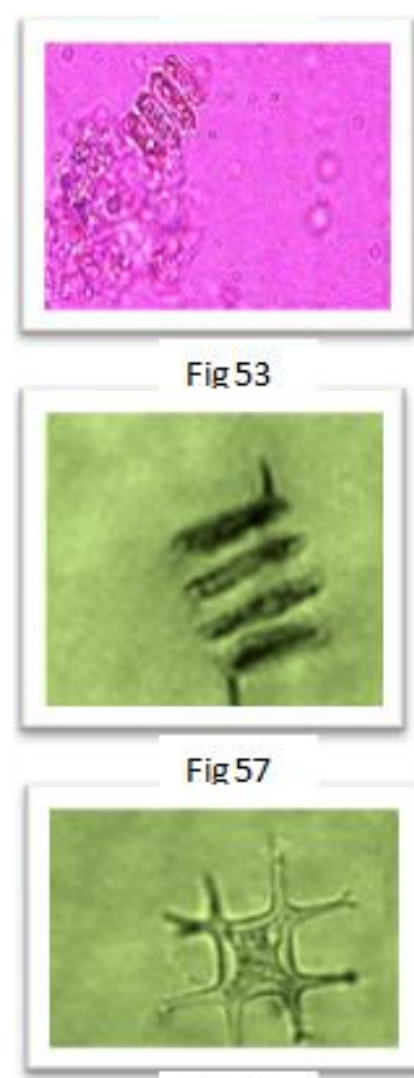

Fig 61
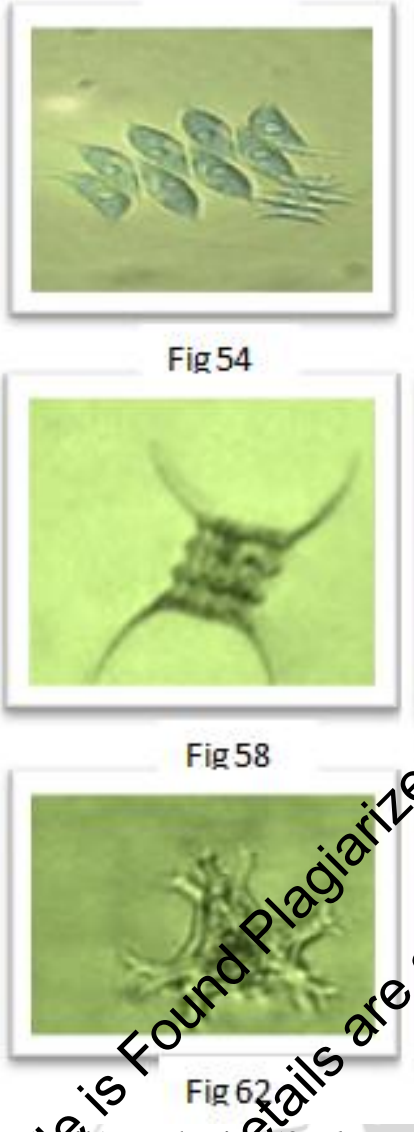

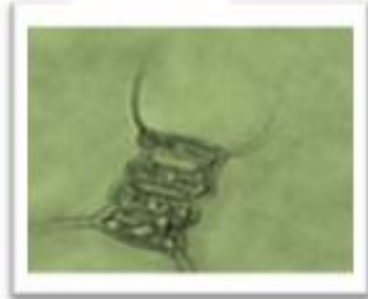

Fig 55
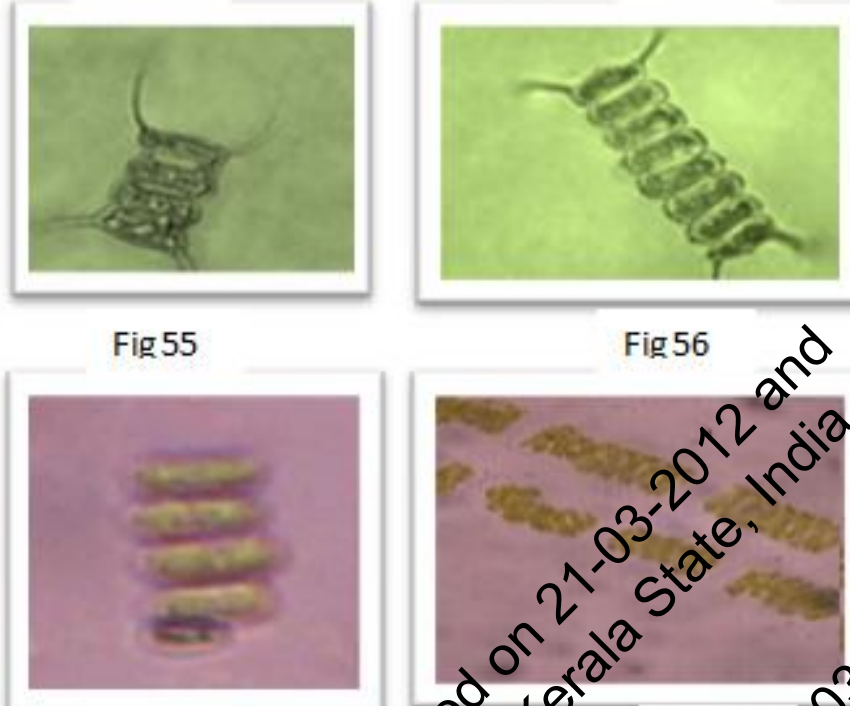

C. Fig 59

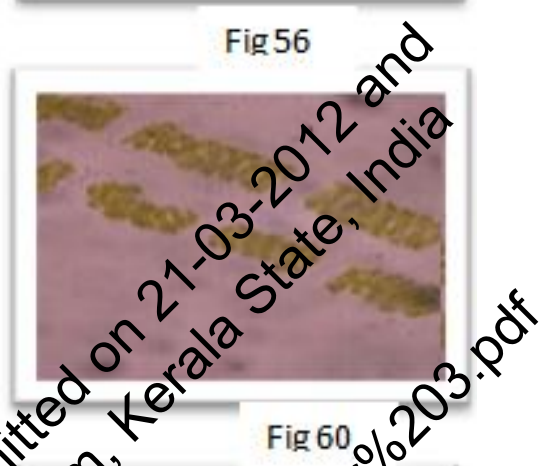

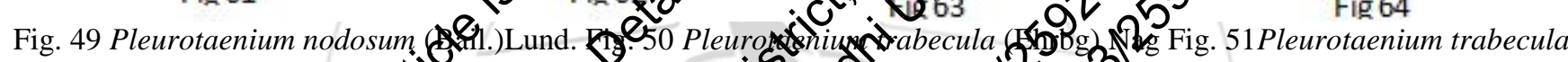

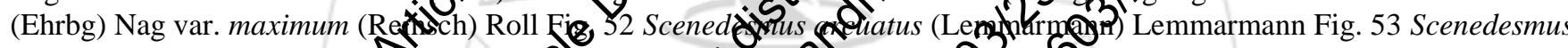
bijugatus (Turp.) Kuetz Fig. Scenedesws dimorphy (Tymat) Kuetzing

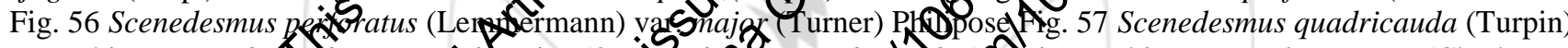

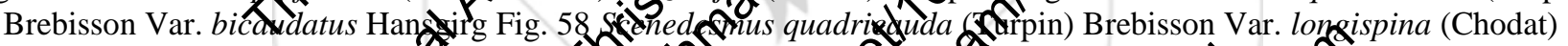

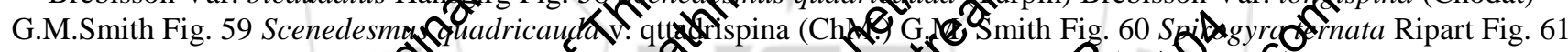

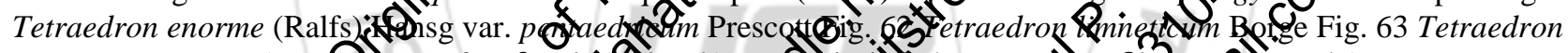

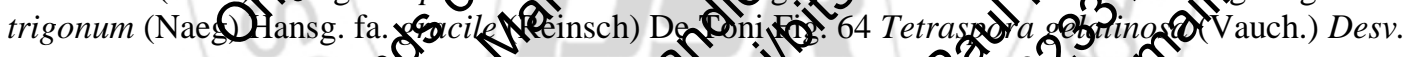

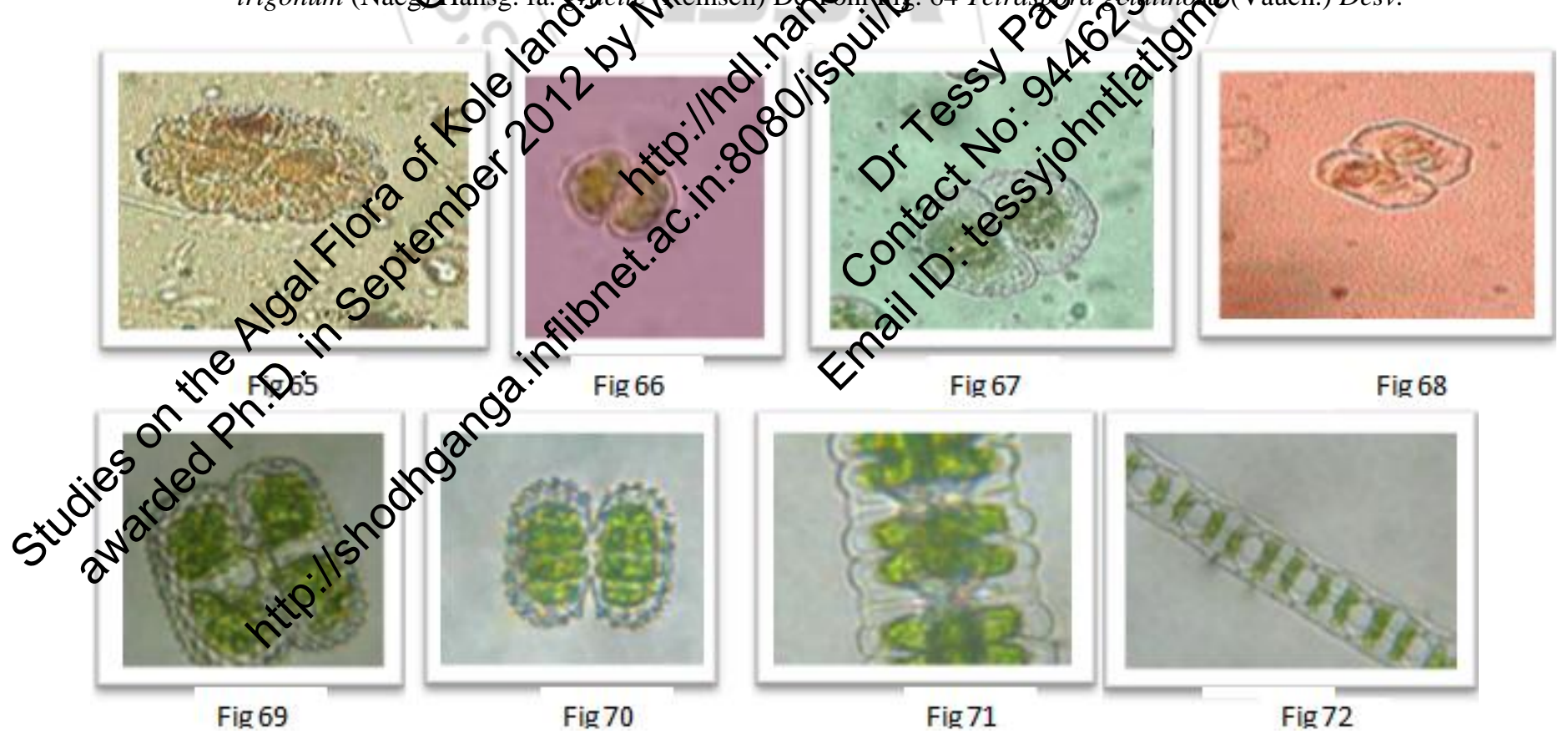




\section{International Journal of Science and Research (IJSR) \\ ISSN (Online): 2319-7064}

Index Copernicus Value (2013): 6.14 | Impact Factor (2014): 5.611

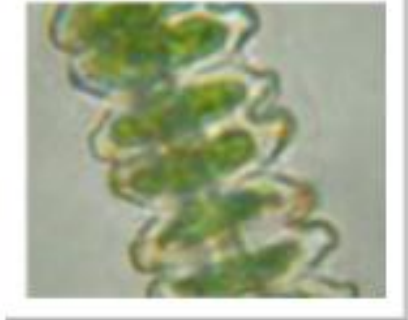

Fig 73

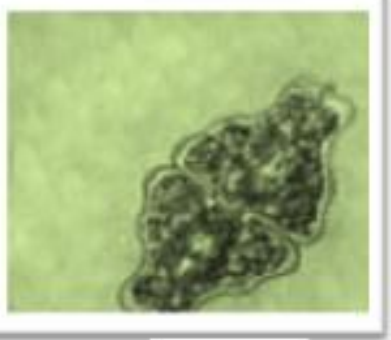

Fig 77

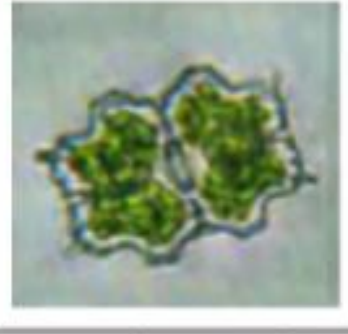

Fig 74

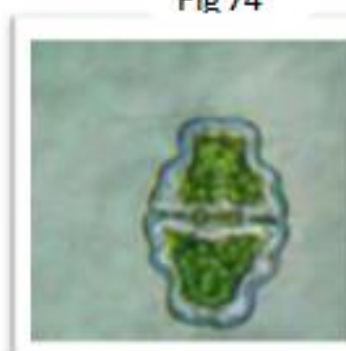

Fig 78

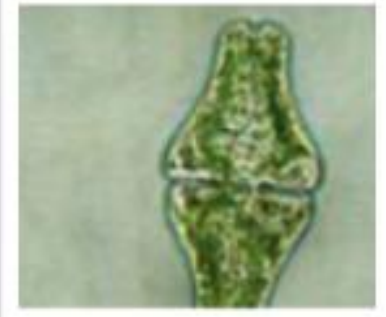

Fig 75
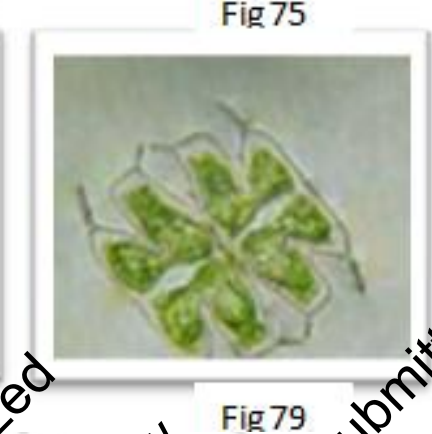

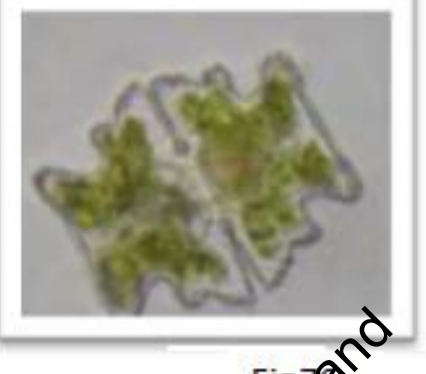

Fig 78

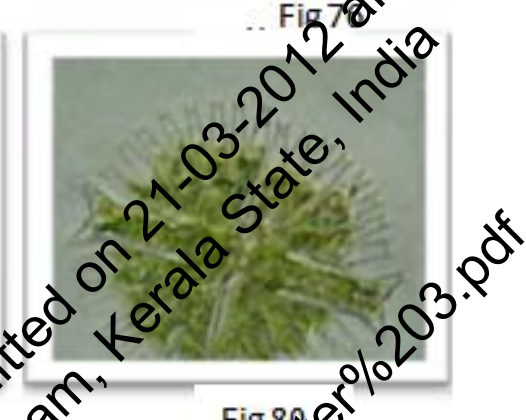

Fig $8 Q \boldsymbol{Q}$

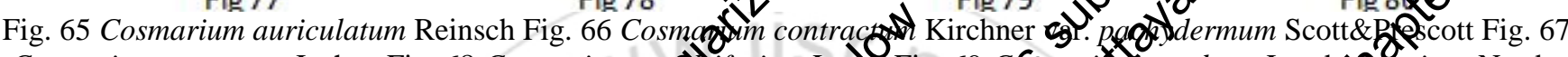
Cosmarium cuneatum Joshua Fig. 68 Cosmarium qyof ifarium LDet. Fig. 69 Gomgrim quadrum Lund in? minus Nordst

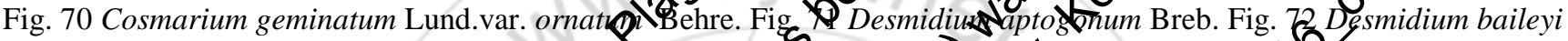
(Ralfs) Nordst. fa. longiprocessum Scott \& Predcott. Fig. 73 Desmidium sgdartzix Agardh. Fig. 74 Eyd Sum acanthophorum

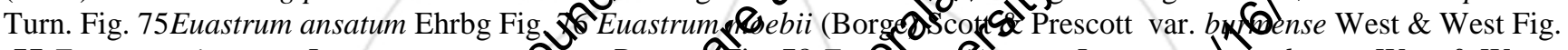

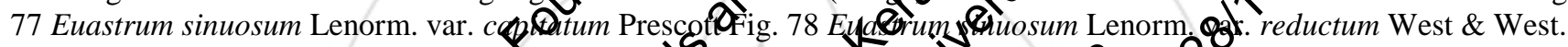

Fig. 79 Micrasterias foliacia Bail væ quadrinflat Pescott Fig. 80 Mifasterias lyposhojw. brevibracchiata Behre fa.
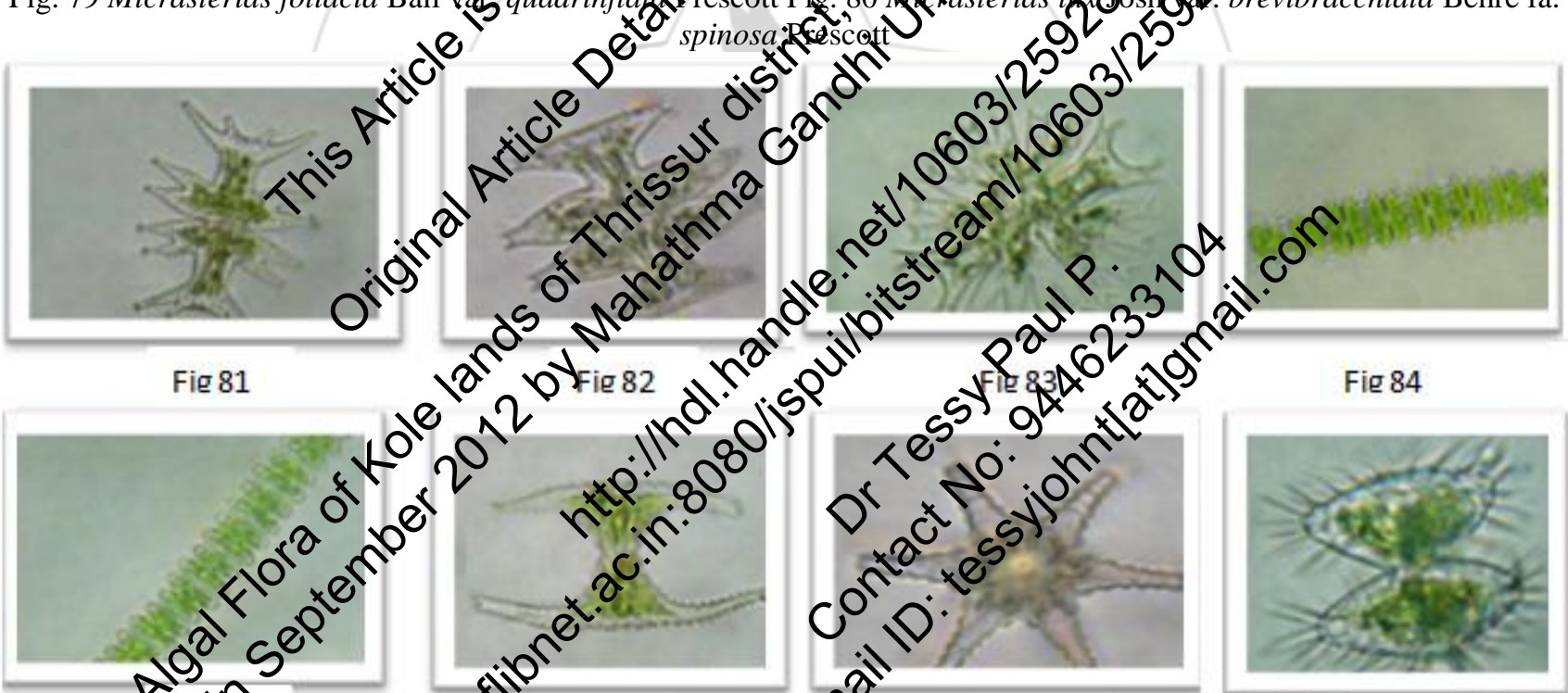

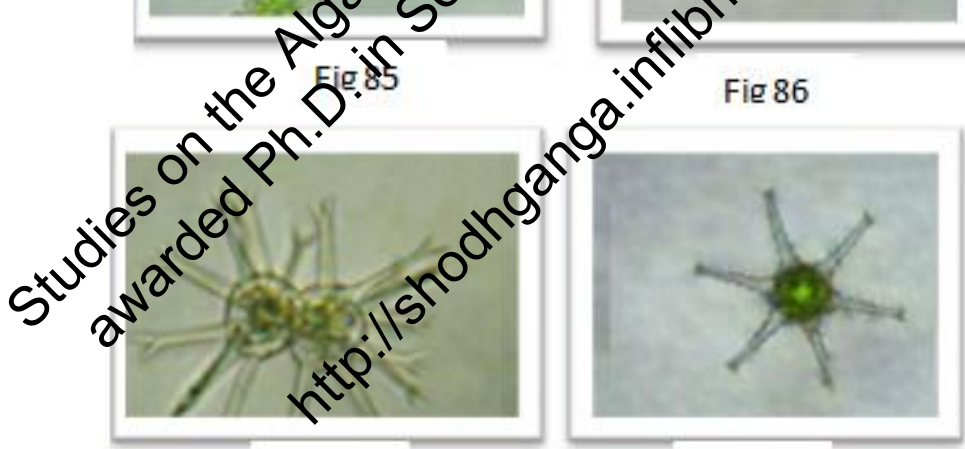

Fig 89

Fig 90

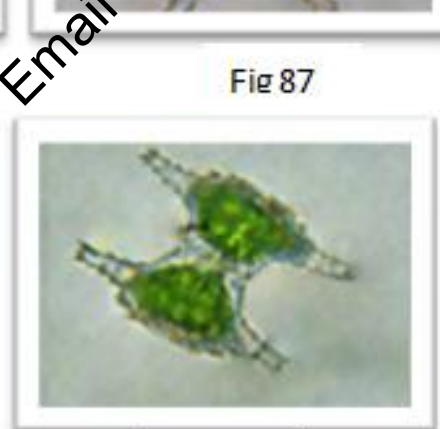

Fig 91
Fig 88

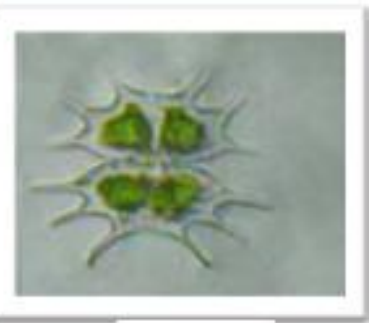

Fig 92 


\section{International Journal of Science and Research (IJSR) \\ ISSN (Online): 2319-7064}

Index Copernicus Value (2013): 6.14 | Impact Factor (2014): 5.611

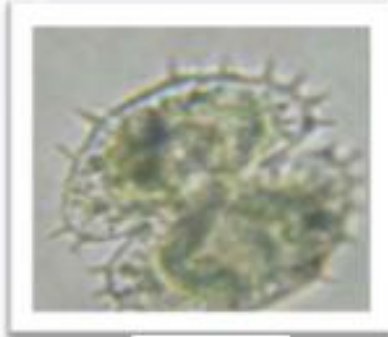

Fig 93

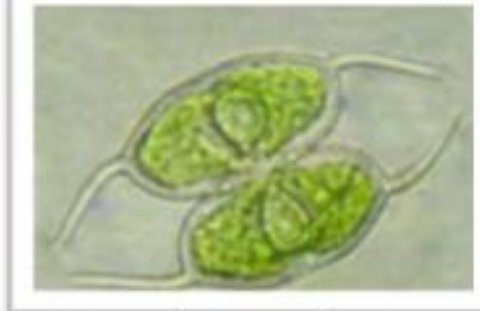

Fig 94

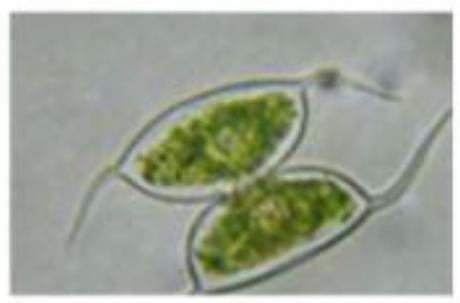

Fig 95

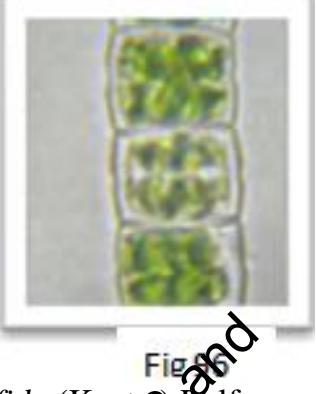

Fig

Fig. 81 Micrasterias mahabuleshwarensis Hobs. var. surculifera Lagerh. Fig. 82 Micrasterias pinnatifida (Kuetr.) Ralffgrar. pseudoscitans Gronbl. Fig. 83 Micrasterias radians Turn. Fig. 84 Onychonema laeve Nordst. var. latum West 8 West $\$ 1 g .85$

Spondylosium planum (Wolle)West \& West. Fig. 86 Staurastrum anatinoides Scott \& Prescott var. javanilaum S\&dt \& Prescott. Fig. 87 Staurastrum pinnatum Turn var. subpinnatum (Sehm)West\&West fa. robustum Krieg \$ig. 8\&\&uarastrum setigerum Cleve.Fig.89. Staurastrum tohopekaligense Wolle var. insigne West \& West. Fig. 90 Staurastrup Xatum Borges

var. majus Presc. Fig. 91 Staurastrum crenulatum (Nag) Delp Fig. 92 Xanthidium sexmamillatunk West $\&$ West var. pulneyense Iyengar \& Bai Fig. 93 Xanthidium. spinosum (Josh.) West \& West Fig. 94 Arthrodesolis ch Mergens Ehr. Fig 92

Arthrodesmus curvatus Turn. var. latus Scott and Prescott Fig. 96 Hyalotheca dissiliens (S_dh) peb. var. hians Wotg?

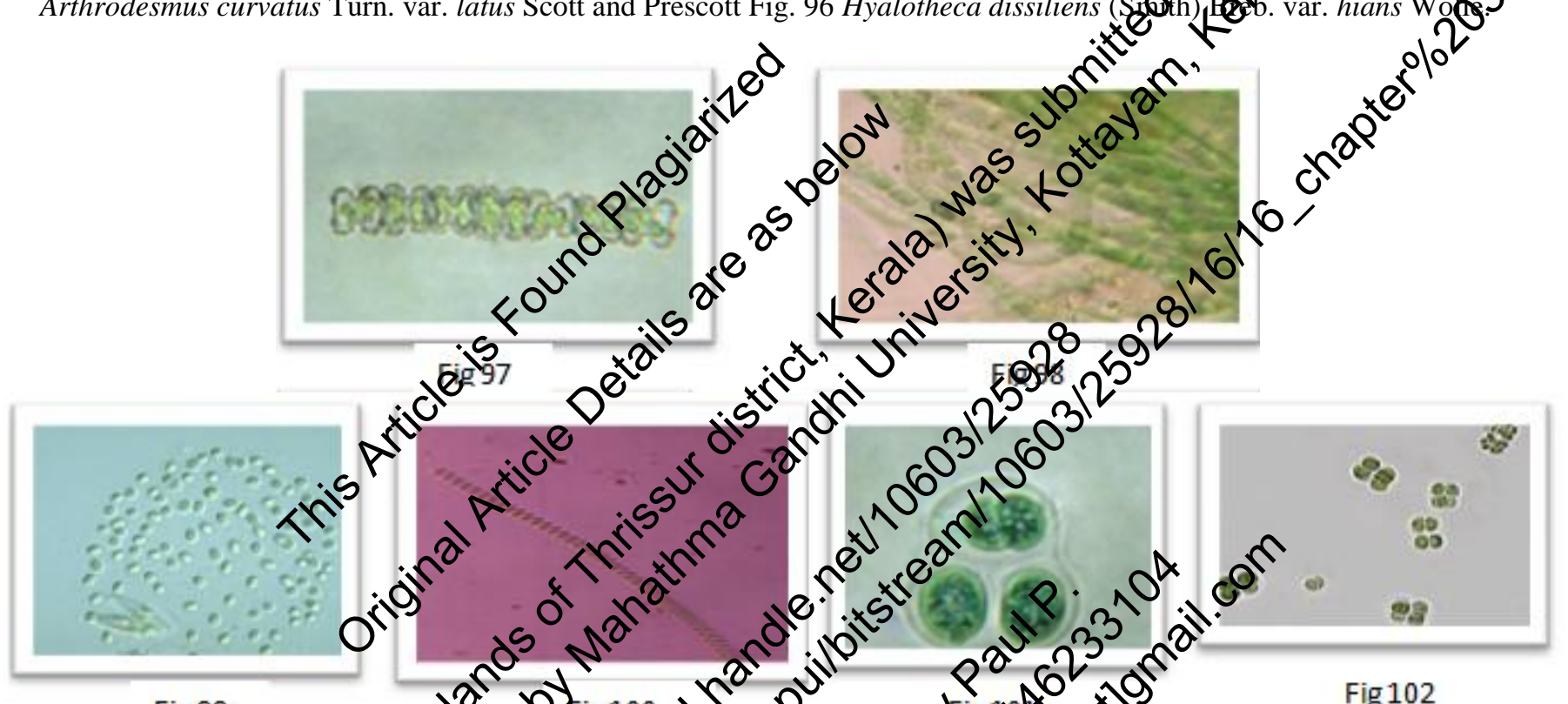

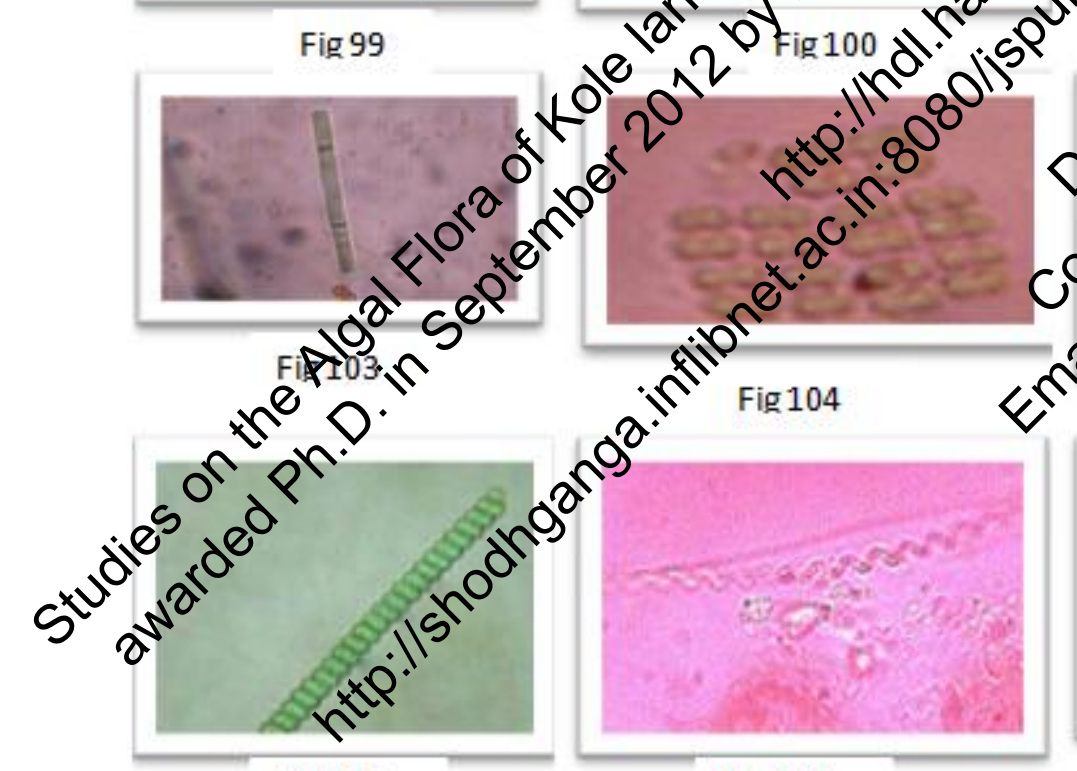

Fig 107

Fie 108
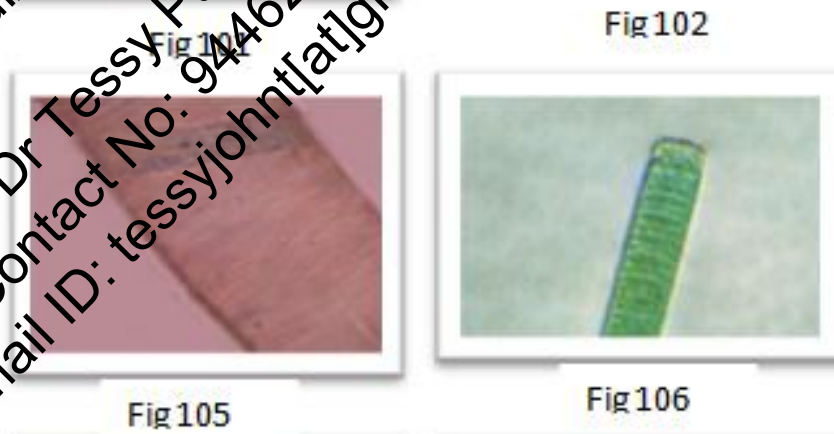

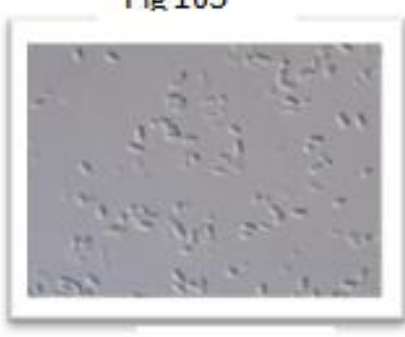

Fig 109
Fig 106

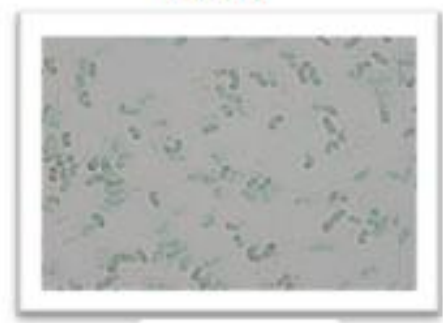

Fig 110

Fig. 97 Sphaerozosma granulatum Roy \& Biss Fig. 98 Anabaena sphaerica var. attenuata Bharadwaja.

Cyanophyceae: Fig. 99 Aphanocapsa littoralis Hansgirg Fig. 96 Arthrospira massartii Kuffareth Fig. 101 Chroococcus turgidus (Kuetz.) Nag. Fig.102 Gleocapsa atrata (Corp.) Kuti Fig.103 Lyngbya aestuarii Liehm. Ex. Gomont Fig.104

Merismopedia glauca (Ehrenb). Nag. Fig.105 Oscillatoria formosa Dory ex Gomont Fig.106 Oscillatoria princeps Vaucher ex Gomont Fig.107 Spirulina labyrinthiformis (Menegh.) Gomont. Fig.108 Spirulina princeps Wet. G.S. West Fig.109 Svnechocvstis aquatilis Sauv. Fig.110 Synechoccus elongates Nag. 


\section{International Journal of Science and Research (IJSR)}

ISSN (Online): 2319-7064

Index Copernicus Value (2013): 6.14 | Impact Factor (2014): 5.611

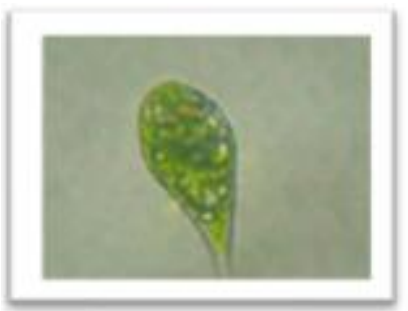

Fig 111

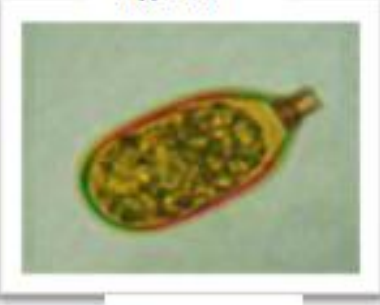

Fig 115

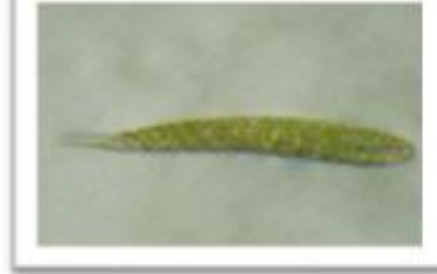

Fig 112

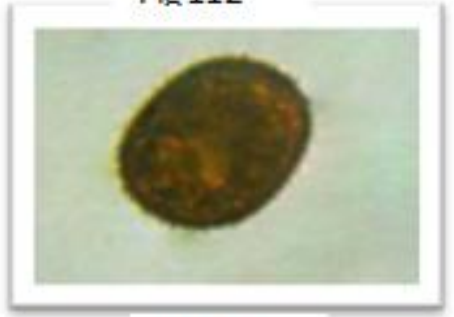

Fig 116

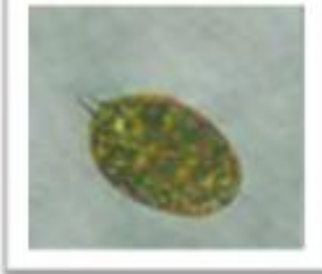

Fig 113

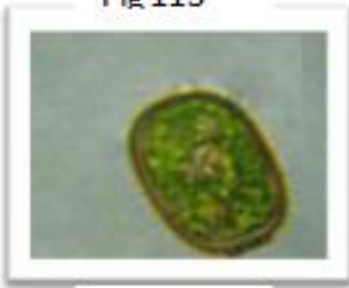

Fig 117

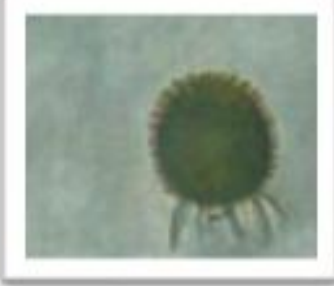

Fig 114

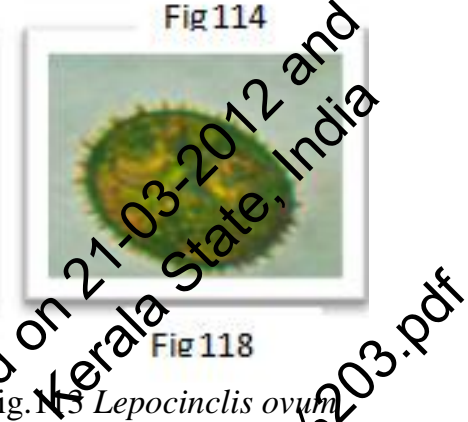

Euglenophyceae Fig.111 Euglena proxima Dangeard. Fig.112 Euglena spirogyra Exig. Lepocinclis ovum

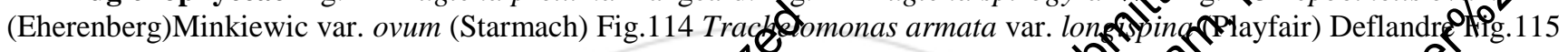

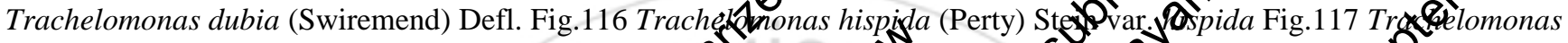

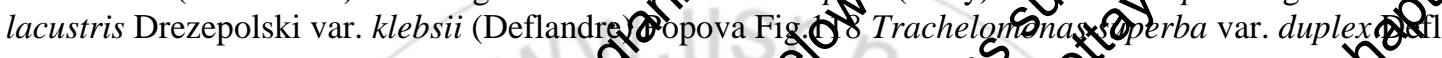

\section{Results and Discussion}

The algal flora of the Vena river in sulganghat comprised 118 taxa belonging to 6K Kenera, asњ was

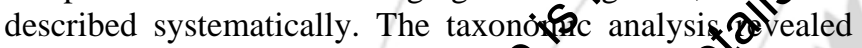
that the phytoplankton of the stux $\ell_{\text {area belgar }}$ to four classes, The classes of $x$ Hilgae regented Bacillariophyceae, Chlorop preae, Cyob (1)phyceae Euglenophyceae, Chlorop (9ceae (gre algae) wos the major group comprised 73 taxa (\$6\%) belon pis to genera. Bacillariophyceae (diatons) was represted taxa (20.33\%) belonging to - 5 genera, Sxglepo hyceae represented by 8 taxa $(6.7$ (0) belonging to 3 enera and Cyanophyceae (blue green algae) remented by 13 tax 8 (11.01\%) belonging to 11 genera noe fople in the study area.

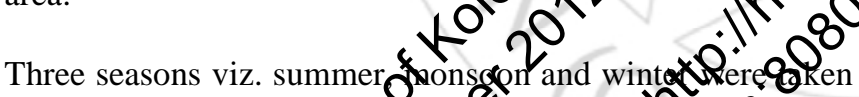

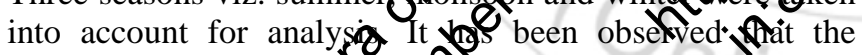
number of phytoplaporons pre maximum dygg winter (408 taxon) followe by during monsoond60 geh).

The number of phytoplanktons recond were analysed by conside statins like $\mathrm{SW}_{1}, \mathrm{~S} 2 \cdot \mathrm{SW}_{3}$, and $\mathrm{SW}_{4}$. . The

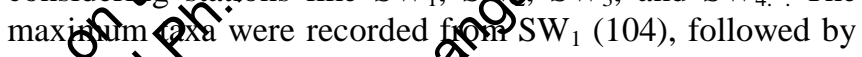
Slos (79d $\mathrm{SW}_{3}(72)$, and lo $5 \mathrm{SW}_{2}$ (68). The maximum \&erd recorded frond $W_{1}$ (51), followed by $\mathrm{SW}_{4}(50)$, SW 43), and lowest $\$ P_{2}$ (42).

$\gamma^{2}$

\section{Acknowledgiments}

Authors would like to thanks Dr. S. N. Malode, Head, P. G. Department of Botany, GVISH, Amaravati and Dr. B. G. Ambatkar, Principal, R. S. Bidkar College, Hinganghat Dist. Wardha for providing the facilities. Authors would also like to thanks Prof. Sebastian Kulthassary for editing the manuscript.

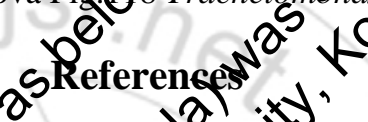

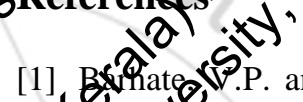

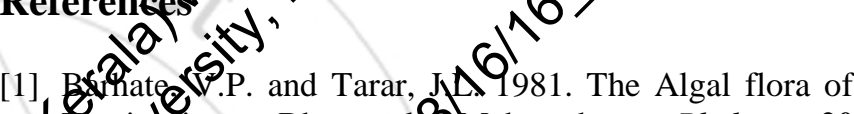
Haptij river, Bousawa 8 Maharashtra. Phykos, 20

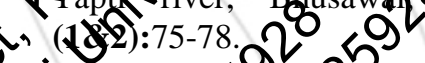

2. Barhate, V and Thrar, J.L. 1983a. Addition to algal Jflora of Atahagatra. Diatoms from Khandesh - I. Phyke O22: $: 50 \%$

[3] Barofe, X. and Tarar, J.L. 1983b. Algae of * aharasta: Addition to Cyanophyceae of Khandesh. Q7.

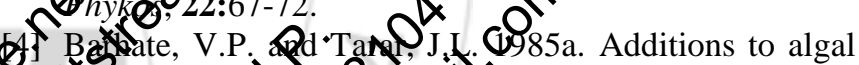
Nora of Mahashtorachlophyceae from Khandesh-I.

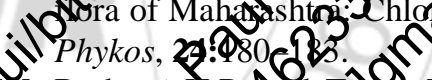

] Barhate V.R Tha florac MQj

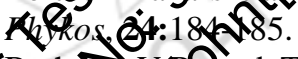

[6]Barbate, V.R Oand Tarar, J.L. 1985c. Additions to the alg(logs Maharashtra: Diatoms from Khandesh - II. Xindi@bot. Soc., 64:381-384.

C7DDzky . L. P. and Nisal, R. S. 2012. Case Study, Diversity - N Fresh Water algae of the Mahakali Dam Wardha, Maharashtra, India AJOBR 0310:1479-1482.

Desikachary, T. V. 1969. Cyanophyta, I.C.A.R., New Delhi.

[9] Desikachary, T. V. 1972. Taxonomy and Biology of Blue-Green Algae. Centre for Advance Study in Botany Madras.

[10] Desikachary, T. V. 1987. Atlas of Diatoms Vol.II, III \& IV. Madras Science Foundation, Chennai.

[11]Desikachary, T. V. 1988. Atlas of Diatoms Vol. V. Madras Science Foundation, Chennai.

[12] Desikachary, T. V., 1959. Cyanophyta, I.C.A.R. monograph on algae, New Delhi. pp. 686.

[13] Nandkar, P.B. 1979. Observations on the algae growing in polluted waters. Ph.D. Thesis, Nagpur University, Nagpur.

[14] Nandkar, P.B., Marathe, K. V. and Motikhaye B. G. 1983 Algae in sewage containing pools and oxidation ponds. Phycos, 22:37. 
[15] Tarar J. L. and Ashan, Jamal 1980. Thermoduric properties of soil algae. J. Indian Soc. Soil Sci., 28:550552.

[16] Tarar, J. L. and Bodkhe, S. 1997. Cyanobacteria from the polluted habitat of Nagpur City. In: Algal Biotechnology. By Trivedi, P. C. Pointer Publishers Jaipur, India. pp.209.

[17] Tarar, J. L. and Bodkhe, S. 1998. Studies on Chlorococcales of Nagpur. Phykos., 37(1\&2): 107-114.

[18] Tarar, J. L., and Seema, Bodkhe 1998. Diatom flora of polluted water bodies of Nagpur.

[19] Tarar, J. L., Charjan, V. and Bodkhe, S. 1998. Contribution to the knowledge of desmids from Nagpur. Phykos., 37(1\&2): 59-67.

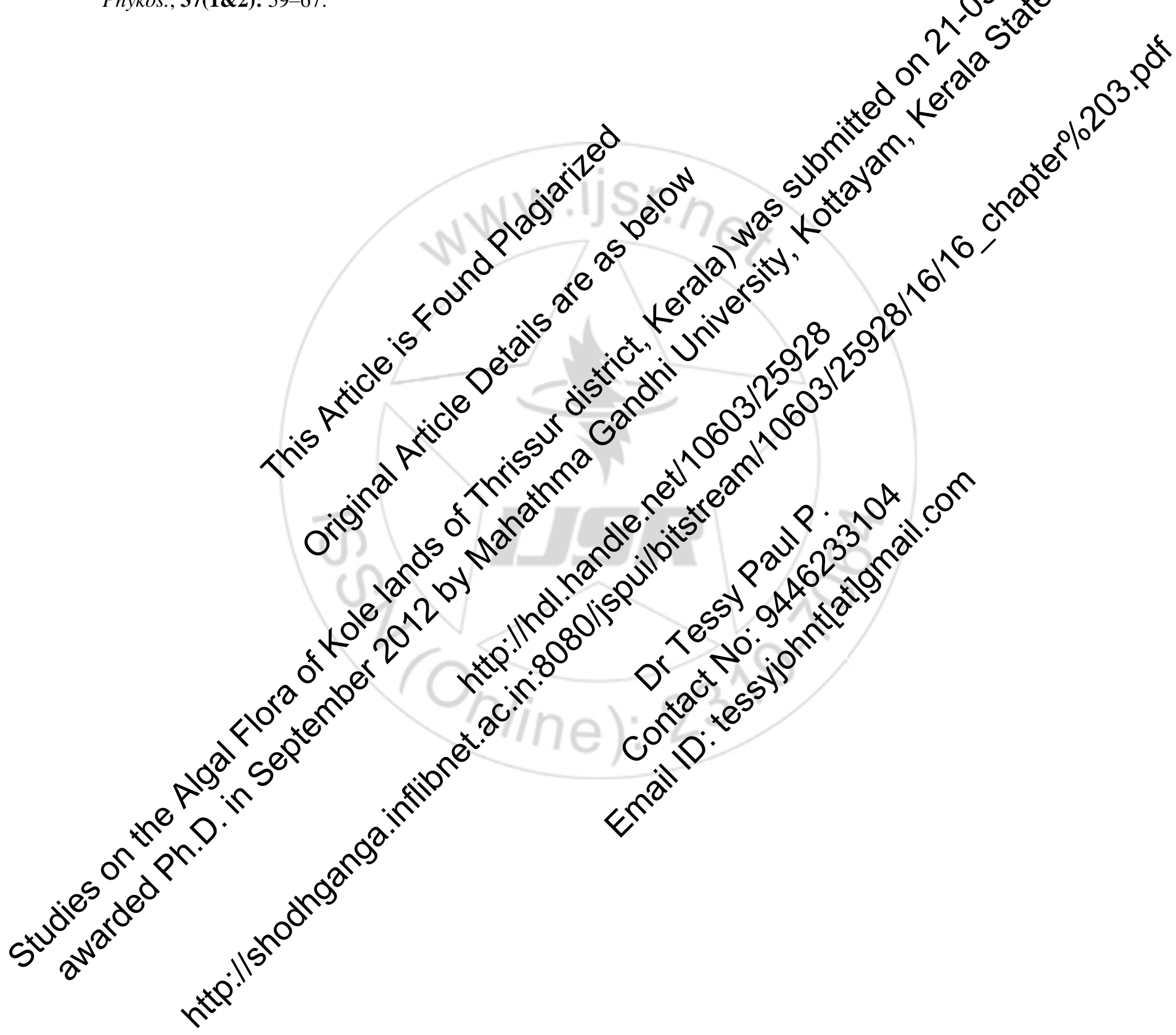

